\title{
Tree-like Shapes Distance Using the Elastic Shape Analysis Framework
}

\author{
Alejandro Mottini ${ }^{1}$ \\ alejandro.mottini_d_oliveira@inria.fr \\ Xavier Descombes ${ }^{1}$ \\ xavier.descombes@inria.fr \\ Florence Besse ${ }^{2}$ \\ Florence.Besse@unice.fr
}

\author{
${ }^{1}$ INRIA CRI-SAM \\ 2004 route des Lucioles, 06902 Sophia \\ Antipolis Cedex, France \\ ${ }^{2}$ IBV \\ Faculté des Sciences, Parc Valrose \\ 06108 Nice Cedex 2, France
}

\begin{abstract}
The analysis and comparison of tree-like shapes is of great importance since many structures in nature can be described by them. In the field of biomedical imaging, trees have been used to describe structures such as neurons, blood vessels and lung airways. Since it is known that their morphology provides information on their functioning and allows the characterization of pathological states, it is of paramount importance to develop methods to analyze their shape and to quantify differences in structures.

In this paper, we present a new method for comparing tree-like shapes that takes into account both topological and geometrical information. It is based on the Elastic Shape Analysis Framework, a framework originally designed for comparing shapes of 3D closed curves in Euclidean spaces. As a first application, we used our method for the comparison of axon morphology. The performance was tested on a group of 44 (20 normal and 24 mutant) 3D images, each containing one axonal tree. We have calculated inter and intra class distances between them and implemented a basic classification scheme. Results showed that the proposed method better distinguishes between the two populations than a pure topological metric. Furthermore, mean shapes can be obtained with this method.
\end{abstract}

\section{Introduction}

Trees are a type of undirected graphs that have no cycles. Therefore, given two nodes, there is only one path between them. In particular, if one of the nodes is labeled root, the tree is called a rooted tree. This type of graphs has been studied thoroughly because, among other things, many naturally occurring structures can be described by them.

In the field of biomedical imaging, some important examples are neurons, blood vessels and lung airways (See Figure 1). It is known that the morphology of these structures provides information on their functioning and allows the characterization of pathological states. Therefore, it is important to have a framework for the analysis of their shapes and to quantify differences in structures.

At present, there are a number of methods for the comparison of trees. One of the best known metrics is TED [四, a method based on the tree-edit distance between unordered labeled tree-graphs. It measures the difference between two trees by counting the number of 

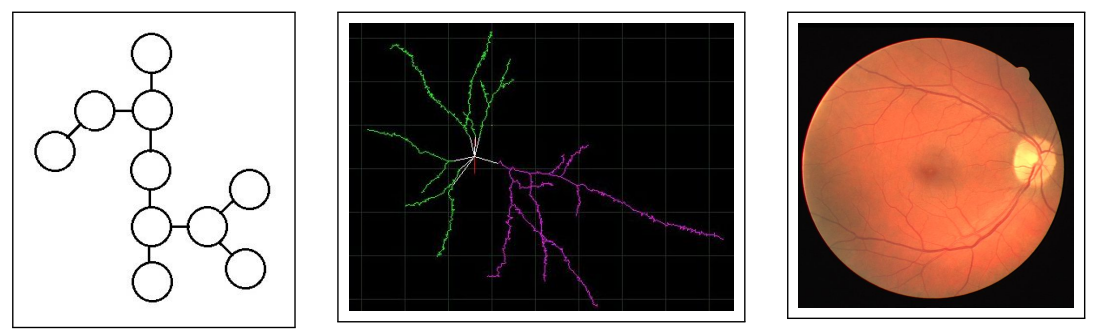

Figure 1: Diagram of a rooted tree (left) and examples of tree-like structure found in nature: neuron (middle) and blood vessels (right). Taken from http://neuromorpho.org/ and http://www.isi.uu.nl/Research/Databases/DRIVE/

nodes that must be added/removed from one tree in order to transform it into the other. This metric has already been successfully applied to neuronal morphology analysis as well as to the analysis of other branching structures such as lung airways. One of the main disadvantages of this method is that double mistakes (an extra branch and a missing one) extending from the same node are ignored. Moreover, the geometry of the curves between nodes is not taken into account.

A different approach was proposed in [ $\square]$. The authors constructed a shape space for rooted treelike shapes and study different metrics on this space. The resulting distance takes into account both topological and geometrical information of the tree. They applied their method to the study of airway trees from pulmonary CT scans. This method presents good results and a strong mathematical foundation.

Other methods have been designed for specific applications, such as the DIADEM metric []. This algorithm was developed as the evaluation method for the DIADEM Challenge and was specifically designed for comparing different tracings of the same neuron. It is therefore not adapted to the comparison of trees in a more general setting.

Finally, Basu et al. [ $[$ ] proposed a metric that takes into account both topological and geometrical information of the shapes. The method is based on finding a deformation energy between two trees. This energy depends (among other things) on the $L_{1}$ norm between the curves that make up the trees. Although the method presents many good ideas, it has a few shortcomings. First of all, the distance is not symmetric. Therefore, their proposed approach does not define a proper metric and is not able to compute geodesic paths between samples or to compute mean shapes. Moreover, the $L 1$ norm is not easy to interpret, as opposed to metrics based on shape spaces, which embed stretching and bending forces necessary to transform one curve into another. In addition, the authors have validated their method by comparing neurons with very different morphologies only (e.g, Pyramidal, Granule and Motor neurons, 2 images for each population).

In this paper, we present a new method for comparing tree-like shapes. The method takes into account both topological and geometrical information, and is specifically designed for analyzing rooted trees that consist of a main curve and several branches (and possibly sub branches) along it. It is based on the Elastic Shape Analysis Framework [ $\mathrm{\theta}]$. We extend this work by defining a new metric between trees.

\footnotetext{
${ }^{1}$ The authors are members of the Morpheme Team (joint INRIA/I3S/IBV research team).

The authors would like to thank Anuj Srivastava and Sebastian Kurtek (Department of Statistics, Florida State University) for providing their original code.
} 


\section{Distance Between Trees}

The proposed method is based on the Elastic Shape Analysis Framework [ $\nabla$ ]. This framework was originally developed to compare shapes of curves in the Euclidean space. The first version was designed to work on closed curves but was later extended to open curves and to include an additional function along with the shape (e.g, a texture function) in the comparison [ $[$ ]. The idea behind this approach is the following. A 3D open curve is parameterized in a special way called SRVF (squared root velocity function). The set of all the SRVF parameterized curves in $\mathbb{R}^{3}$ defines a manifold called the preshape space. In order to only compare the shape of the curves, all shape preserving transformations are removed from the representation (translation, rotation, scale and parametrization). This forms a quotient space of the original manifold called the shape space. Finally, one can define geodesics in the resulting space that can later be used to define distances and to compute averages of sets of curves.

\subsection{Elastic Shape Analysis Framework}

Let the main curve and each branch (and sub branch) of the tree be represented by a parameterized curve $\beta:[0,1] \rightarrow \mathbb{R}^{3}$. Curve $\beta$ is later reparameterized using its $\operatorname{SRVF} q(t)=\frac{\beta \dot{(t)}}{\sqrt{\|\beta(t)\|}}$, where $\|x\|$ is the standard Euclidean norm in $\mathbb{R}^{3}$. Since $q$ depends on the time derivative of $\beta$, the analysis will be independent of the position of the curve (obtaining a translation invariant representation). The authors later rescale the curves to length one in order to impose scale invariance.

We define the preshape space $\mathcal{C}$ as follows: $C=\left\{q:[0,1] \rightarrow \mathbb{R}^{3}\right\}$.

However, curves that differ by a rotation/reparameterization result in different points in the preshape space despite having the same shape. This is solved by removing the last two remaining shape preserving transformations: rotation and reparameterization.

Let $S O(3)$ be the group of $3 \times 3$ rotation matrices and $\Gamma$ be the group of all reparameterizations. Given a curve $\beta$ with $\operatorname{SRVF} q$, a rotation $O \in S O(n)$ and a re-parametrization $\gamma \in \Gamma$, the transformed curve can be expressed as: $q^{\star}=\sqrt{\dot{\gamma}} O(q \circ \gamma)$.

All the elements in $\mathrm{C}$ that represent the same shape can be unified by defining the equivalence class:

$$
[q]=\{O(q \circ \gamma) \sqrt{\dot{\gamma}} \mid O \in S O(n), \gamma \in \Gamma\}
$$

Each class $[q]$ defines an unique shape and the set of all these equivalence classes is called the shape space $S$. This new space is actually a quotient space of the preshape space: $S=$ $C /(S O(n) \times \Gamma)$.

The authors then impose a Riemannian structure on the shape space by imposing the $L^{2}$ norm on its tangent spaces, which corresponds to assuming that the curves and their derivatives are square integrable. This allows the computation of geodesics between points in $S$. The distance between two points in $S$ is defined as the length of the geodesic between them. Moreover, the geodesic can be interpreted as the optimal elastic deformation from one curve into the other.

Given two curves $\beta_{1}, \beta_{2}$ represented by their corresponding SRVF $q_{1}, q_{2}$, the first step for computing the geodesic between the two is fixing $q_{1}$ and finding the optimal rotation and parametrization of $q_{2}$ such that:

$$
\left(O^{*}, \gamma^{*}\right)=\underset{O \in S O(3), \gamma \in \Gamma}{\arg \min }\left\|q_{1}-\sqrt{\dot{\gamma}} O\left(q_{2} \circ \gamma\right)\right\|^{2}
$$




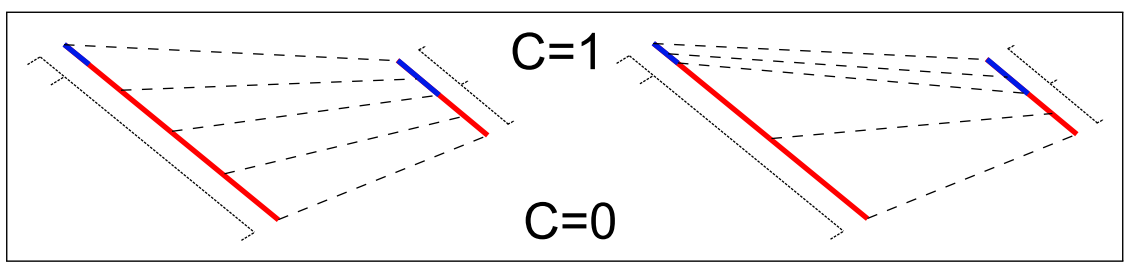

Figure 2: Registration between two curves including a simple step $C$ function (blue for $C=1$, red for $C=0$ ), $b=0$ (left) and $b=\infty$ (right). If $b$ is big the registration is mostly determined by the $C$ function.

The optimization over rotation can be achieved with SVD, while the optimization over the reparametrization requires dynamic programming. We can then define:

$$
q_{2}^{*}=\sqrt{\gamma^{*}} O^{*}\left(q_{2} \circ \gamma^{*}\right)
$$

The geodesic path between $q_{1}$ and $q_{2}^{*}$ is given by:

$$
\alpha(\tau)=(1-\tau) q_{1}+\tau\left(O^{*}\left(q_{2}, \gamma^{*}\right)\right)
$$

while the distance between the two curves is given by:

$$
d_{\text {shape }}\left(\beta_{1}, \beta_{2}\right)=\min _{\gamma \in \Gamma, O \in S O(3)}\left\|q_{1}-O(q 2, \gamma)\right\|_{2}
$$

An important consequence of this method is that at the end, there is a matching of points along the two curves (see Figure 2). This property will later be used to calculate the transformation between two axonal trees.

The previous framework was extended in [日] to include additional information in the shape analysis. This is achieved by including an additional function $\beta_{c}:[0,1] \rightarrow \mathbb{R}^{k}$ (where $k$ is an arbitrary dimension) to the original representation of curve $\beta$. The two components are combined to form a new curve:

$$
\beta(t)=\left[\begin{array}{c}
\beta_{s} \\
b . \beta_{c}
\end{array}\right] \in \mathbb{R}^{3+k}
$$

where $b>0$ is a control parameter. The remaining steps are done in the same way as before but with the new curve $\beta$ (although special precaution not to rotate the $\beta_{c}$ component are required when eliminating the rotation transformation).

\subsection{Metric Formulation: From Curves to Trees}

In this section, we extend the Elastic Shape framework to define a metric between trees. We consider two trees $T_{1}$ and $T_{2}$, each consisting of a main curve and several branches (and possibly sub branches). All are represented by $3 \mathrm{D}$ open curves in $\mathbb{R}^{3}$. Each branch forms an angle $\phi \in[0, \pi]$ with respect to its mother branch (the main curve in the case of first level branches) and an angle $\theta \in[0,2 \pi)$ with respect to the first branch of its mother branch (see Figure 3).

We start by defining the matching function $M$ such that $M:(0,1,2, \ldots n) \times(0,1, \ldots, m)$, where $n$ and $m$ are the number of branches in $T_{1}$ and $T_{2}$ respectively. The matching function matches the branches of the two trees as follows:

$$
M(i, j)= \begin{cases}1 & \text { if } \mathrm{i} \text { matches } \mathrm{j} \\ 0 & \text { otherwise }\end{cases}
$$




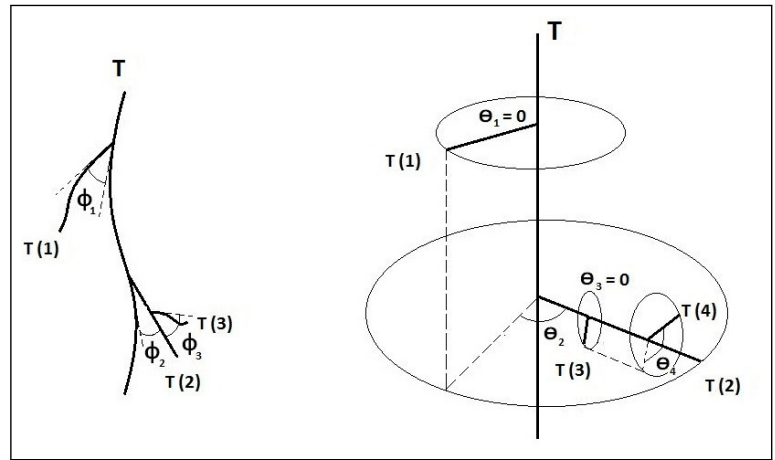

Figure 3: Tree diagrams showing how angles $\phi$ and $\theta$ are defined for a given branch. Angles $\phi_{1}$ and $\phi_{2}$ are defined with respect to the main branch, while $\phi_{3}$ is defined with respect to $T$ (2) (its mother branch). A similar convention is taken for $\theta$. In this example, the angle $\theta_{2}$ associated to branch $T(2)$ is measured with respect to branch $T(1)$, while $\theta_{4}$ is measured with respect to $T(3)$ (the first branch depending from its mother branch).

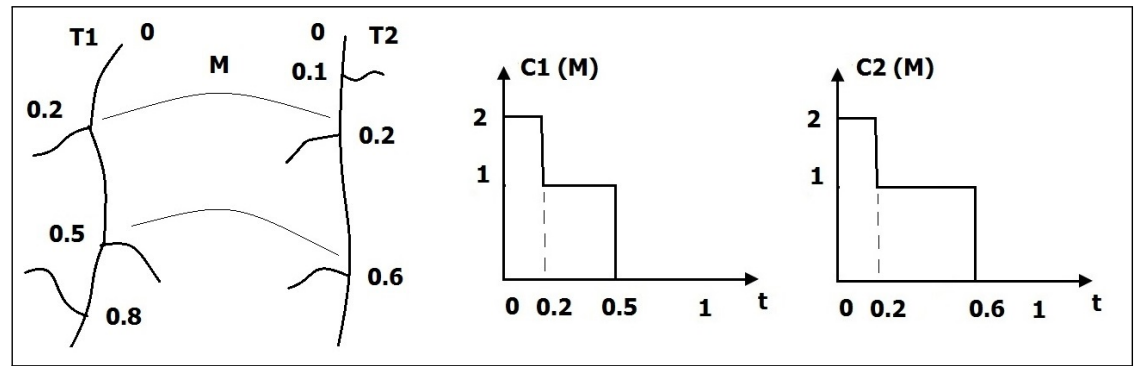

Figure 4: Tree diagrams (left) and their corresponding $C$ functions for a given $M$ (right).

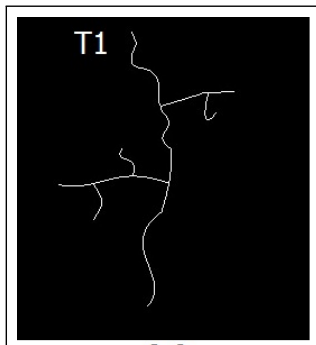

(a)

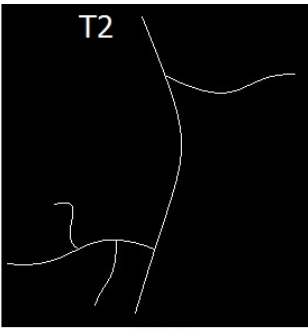

(b)

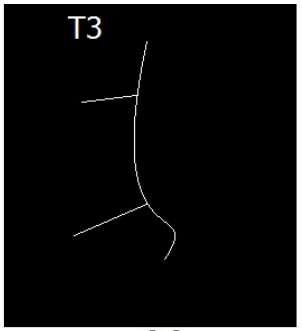

(c)

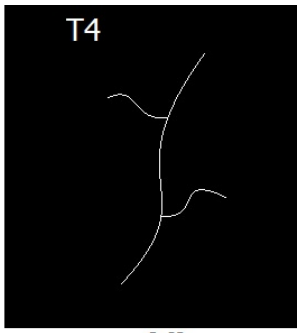

(d)

Figure 5: Four synthetic images each containing a simple tree with a main curve and some branches and sub branches. 
To improve the efficiency of the algorithm, we do not allow the matching of branches that are far away from each other. Other constraints are also included, such as not allowing the crossed matching of branches (e.g, matching branches 1 and 2 of $T_{1}$ to branches 2 and 1 of $T_{2}$ ).

We then define a branch function $C$ which indicates, for a given time $t_{c}$, how many branches remain after $\beta\left(t_{c}\right)$ (see Figure 4$)$. We only take into account branches which have a match in the other tree.

$\mathrm{C}$ functions are defined for both trees and included as the fourth component of $\beta_{1}, \beta_{2}$ as explained in the previous section. This allows us to take into account the difference of position of matched branches in the distance between trees.

Finally, we define the distance between two trees $T_{1}, T_{2}$ as:

$$
\begin{array}{r}
D\left(T_{1}, T_{2}\right)=\min _{M}\left[d\left(\left(\beta_{1}(t), C 1(t, M)\right),\left(\beta_{2}(t), C 2(t, M)\right)\right)+\right. \\
\left.+\sum_{(i, j)} \alpha_{i, j} M(i, j) D\left(T_{1}(i), T_{2}(j)\right)\right]
\end{array}
$$

where $\beta_{k}$ is the main curve of tree $k, C_{k}$ its branch function, $M$ the matching function, $\alpha_{i, j}$ a weight parameter and $D\left(T_{1}(i), T_{2}(j)\right)$ the distance between the matched branches (sub trees) of the two trees.

The first term of the distance definition embeds some geometrical information on the main branch by matching segments between branching points. Topological information is taken into account through the $C$ function. The parameter $b$ in equation 6 weights the two characteristics. Sub branches are taken into account in a recursive way in the second term. Note that the coefficients weight the different order of sub branches with respect to the main branch. Moreover, $\alpha_{i, j}$ controls how non matched branches $(M(i, 0)=1$ or $M(0, j)=1)$ are penalized. Typically, $\alpha_{i, j}$ equals $a_{1}$ if $i \neq 0, j \neq 0$ and it equals $a_{2}$ otherwise.

The distance between the two main curves is defined as:

$$
\begin{array}{r}
d\left(\left(\left(\beta_{1}(t), C 1(t, M)\right),\left(\beta_{2}(t), C 2(t, M)\right)\right)=d_{\text {length }}\left(\beta_{1}(t), \beta_{2}(t)\right)+\right. \\
\left.+w_{1} d_{\text {shape }}\left(\beta_{1}(t), C 1(t, M)\right),\left(\beta_{2}(t), C 2(t, M)\right)\right)
\end{array}
$$

where $d_{\text {length }}$ is defined as:

$$
d_{\text {length }}\left(\beta_{1}(t), \beta_{2}(t)\right)=\frac{\| \text { length }\left(\beta_{1}(t)\right)-\text { length }\left(\beta_{2}(t)\right) \|}{\| \text { length }\left(\beta_{1}(t)\right)+\operatorname{length}\left(\beta_{2}(t)\right) \|}
$$

and $d_{\text {shape }}$ is defined by equation 5 . The parameter $w_{1}$ controls the weight between the two terms.

In addition, the distance between two matched branches is defined as:

$$
\begin{array}{r}
D\left(T_{1}(i), T_{2}(j)\right)=d_{\text {length }}\left(\beta_{i}(t), \beta_{j}(t)\right)+w_{1} d_{\text {shape }}\left(\left(\left(\beta_{i}(t), C i(t, M)\right),\left(\beta_{j}(t), C j(t, M)\right)\right)+\right. \\
+w_{2} d_{\phi}\left(\phi_{i}, \phi_{j}\right)+w_{3} d_{\theta}\left(\theta_{i}, \theta_{j}\right)
\end{array}
$$

where $d_{\text {length }}$ and $d_{\text {shape }}$ are defined in the same way as for the main curve, and the two remaining terms as:

$$
\begin{array}{r}
d_{\phi}\left(\phi_{1}, \phi_{2}\right)=\left\|\phi_{1}-\phi_{2}\right\| / \pi \\
d_{\theta}\left(\theta_{1}, \theta_{2}\right)=\left\|\theta_{1}-\theta_{2}\right\| / 2 \pi
\end{array}
$$




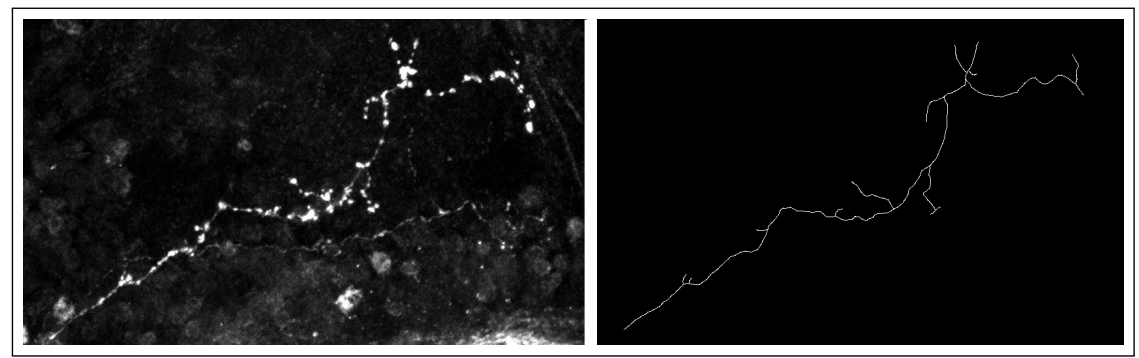

Figure 6: Original confocal microscopy image of a normal axonal tree (left) and its manual tracing (right) (maximum intensity projections).

Once again, the parameters $w_{i}$ control the weight between the terms.

Finally, the distance for a branch with no match is defined as:

$$
D\left(T_{1}(i), 0\right)=w_{\text {kill }} \text { length }\left(\beta_{i}(t)\right)
$$

where $w_{\text {kill }}$ is a parameter of the algorithm and $\beta_{j}(t)$ is the curve associated to the mother branch of $T_{1}(i)$.

The geodesic defined by equation 4 is adapted to simple curves. To uniquely define the geodesic between trees, we consider that the transformations between matched branches and the creation/removal of branches occur uniformly during the same time interval $[0,1]$.

To illustrate how the different parameters can alter the matching of the branches and the final results, let us consider a set of simple synthetic images (see Figure 5). Let us first considers images (a) and (b). If the parameters are chosen in a given way, similar branches and sub branches should match each other, that is, $T 1(i)$ should match $T 2(i)$. In this case, branch $T 1$ (3) will have no match (it would be matched to 0 , thus being "killed"). However, if we tune the parameters in a different way, this result could change significantly. For example, if the cost of killing a branch is relatively small compared to transforming one branch into another, we would be in a scenario where all the branches of $T 1$ are killed and all the branches of $T 2$ are being born (all branches matched to 0 ). On the other hand, if we consider images (c) and (d), we can illustrate the effect of $w_{3}$ on the matching. For a set of parameters where $w_{3}$ is relatively small with respect to the other terms, one would expect $T 3$ (2) to match $T 4(2)$. However, if we increase the weight of $d_{\theta}$ in the total distance, the matching of these two branches becomes too expensive as it requires the rotation of branch $T 3$ (2) by $\pi$. In this case, the method would match both branches to 0 .

\section{Application: Axon Morphology Comparison}

Much effort has been put in recent years into the analysis of neuronal morphology. It is known that it impacts network connectivity, thus providing information on its functioning. Moreover, it allows the characterization of pathological states. Therefore, the analysis of the morphological differences between normal and pathological structures is of paramount importance. In particular, the analysis of neuronal axonal topologies allows biologists to study the causes of neurological diseases such as Fragile X Syndrome or Spinal Muscular Atrophy [0]. 


\subsection{Validation}

For the validation, we have labeled single neurons within intact adult Drosophila fly brains and have acquired 3D fluorescent confocal microscopy images of their axonal trees. Both normal neurons and neurons in which a gene related to neurological diseases in humans was inactivated have been imaged. Each image stack contains a single axonal tree and has a resolution of $0.093967 \times 0.093967 \times 0.814067 \mu \mathrm{m}$. Later, these images were manually segmented by an experienced biologist (see Figure 6) and the result converted to 3D open curves in $\mathbb{R}^{3}$. In total, 44 images (20 normal and 24 mutant) were used.

\subsection{Results}

For the validation of our method we have compared our results with the ones obtained by RTED [ $[$ ], an efficient solution for the tree edit distance problem. The inter and intra class distances were calculated using all the images in our database. The mean and standard deviation of these distances are presented in Table 1. The reported average intra-class distance is the average over all possible pairs of trees within each class, and the average inter-class distance is the average over all possible pairs of trees with one tree from each class. Results show that the proposed method is able to distinguish between the two populations, with an average interpopulation to intrapopulation distance ratio of 1.3 and 1.6 (see Table 2). Moreover, we looked at the nearest neighbors of each image in the database (see Table 3) and we have embedded our metric in a basic classification method that we used to separate the two populations (see Table 4). The classification method is based on K-Means. We start with two centroids randomly chosen among the total set of samples. We assign each sample to the cluster whose centroid is closest to it. Finally, we calculate the new centroid of each cluster to be the observation that has the minimum total intra distance (instead of calculating the cluster mean as in K-Means).

Altogether, these results we obtained are far better than those obtained with the TED method for the normal samples, and a bit worst than those obtained with the TED method for the mutant samples. Therefore, we believe our method obtains a better overall performance. Notably, neither method is able to separate perfectly the two classes, which can be explained by the fact that in our image database, samples from the two analyzed populations are similar in some cases (see Figure 7).

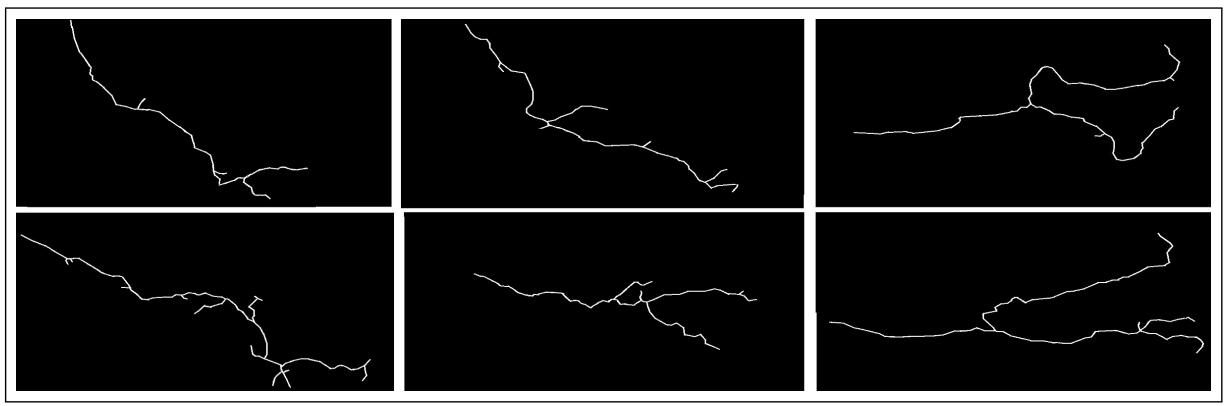

Figure 7: Some examples of mutant (top) and normal (bottom) axonal trees that are similar to each other (2D projections). 


\begin{tabular}{|c|c|c|}
\hline Population (Method) & Mean & Std \\
\hline \hline Normal (ESA) & 1615.5 & 660.6 \\
\hline Mutant (ESA) & 1348.7 & 695.0 \\
\hline Inter Population (ESA) & 2092.4 & 796.4 \\
\hline Normal (RTED) & 260.2 & 97.5 \\
\hline Mutant (RTED) & 98.8 & 56.6 \\
\hline Inter Population (RTED) & 195.6 & 97.2 \\
\hline
\end{tabular}

Table 1: Mean and Std of the inter and intra population distances for the proposed method (ESA) and RTED.

\begin{tabular}{|c|c|}
\hline Populations (Method) & Ratio \\
\hline \hline Inter / Normal (ESA) & 1.3 \\
\hline Inter / Mutant (ESA) & 1.6 \\
\hline Inter / Normal (RTED) & 0.8 \\
\hline Inter / Mutant (RTED) & 2.0 \\
\hline
\end{tabular}

Table 2: Intra and inter population distance ratios for our method (ESA) and RTED.

\begin{tabular}{|c|c|c|c|}
\hline Population (Method) & 1 & 2 & 3 \\
\hline \hline Normal (ESA) & $75 \%$ & $75 \%$ & $80 \%$ \\
\hline Mutant (ESA) & $88 \%$ & $88 \%$ & $83 \%$ \\
\hline Normal (RTED) & $11 \%$ & $5 \%$ & $16 \%$ \\
\hline Mutant (RTED) & $92 \%$ & $100 \%$ & $100 \%$ \\
\hline
\end{tabular}

Table 3: Percentages of axons that have a nearest neighbor that belongs to the same population (from first to third neighbor), when using ESA and RTED.

\begin{tabular}{|c|c|c|c|}
\hline Method & Well Classified - Normal & Well Classified - Mutant & Overall Accuracy \\
\hline \hline ESA & $85 \%$ & $91.7 \%$ & $88.6 \%$ \\
\hline RTED & $52.6 \%$ & $79.2 \%$ & $67.4 \%$ \\
\hline
\end{tabular}

Table 4: Percentage of well classified axons for each population and overall accuracy for ESA and RTED.

Importantly, our method allows us to compute mean shapes between two axonal trees by taking the middle point along the geodesic between the two trees (Figure 8). This is something that TED and other methods are unable to do. Having the mean shape will allow us to derive more complex classification scheme such as the K-means algorithm.

\section{Conclusions}

A method for the comparison of tree-like structures embedding geometrical and topological properties was proposed. This method is an extension of the Elastic Shape Analysis Framework, which was originally developed for simple curves. The distance between two structures is defined as the length of the geodesic between them in a space of tree-like shapes. Moreover, our method is able to show how one tree transforms into the other (by taking intermediate points along the geodesic) and to compute the mean shape between them.

As a first application, we used our method for the comparison of axon morphology. The performance was tested on a group of 44 (20 normal and 24 mutant) 3D images, each containing one axonal tree manually segmented by an experienced biologist from a set of real confocal microscopy images. We have calculated the mean and standard deviation of the inter and intra class distances between the neurons and implemented a basic classification 


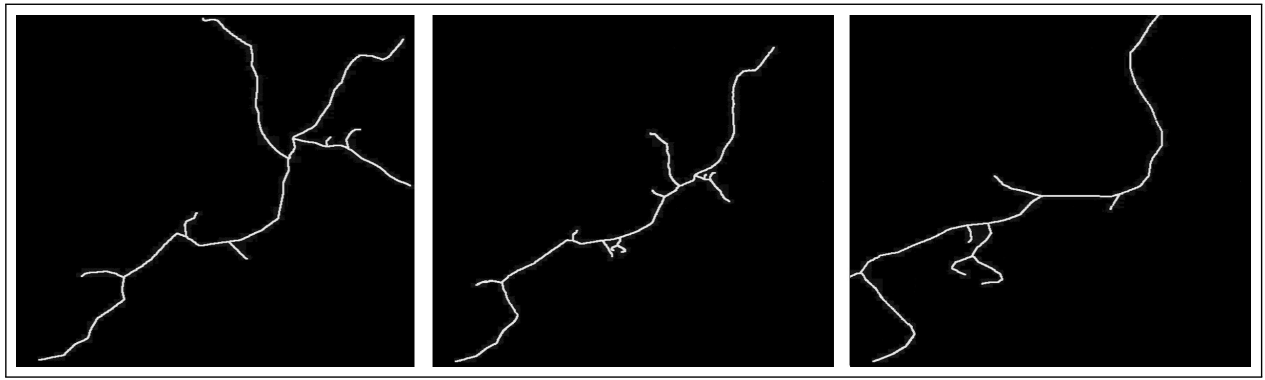

Figure 8: Two axonal trees (left and right) and their mean shape (middle) (2D projections).

scheme. We determined that the proposed method is able to distinguish between the two populations. Moreover, we have compared our results with the ones obtained by TED and concluded that our method obtains a better overall performance.

In the future, we intend to improve the quantitative evaluation of the method and to apply it to other tree like structures. In addition, we plan on proposing an algorithm for computing the average shape of a population and to develop a statistical framework for analyzing and classifying different populations.

\section{References}

[1] H. Heumann and G. Wittum. The tree-edit-distance, a measure for quantifying neuronal morphology. Neuroinformatics, 7(3):179-190, 2009.

[2] A. Feragen, F. Lauze, P. Lo, M. de Bruijne, and M. Nielsen. Geometries on spaces of treelike shapes. In Proc. 10th Asian conference on Computer vision, Volume Part II, 160-173, 2010.

[3] T. A. Gillette, K. M. Brown, and G. A. Ascoli. The diadem metric: comparing multiple reconstructions of the same neuron. Neuroinformatics, 9(2,3):233-245, 2011.

[4] S. Basu, B. Condron, and S. T. Acton. Path2path: Hierarchical path-based analysis for neuron matching. In Proc. IEEE International Symposium on Biomedical Imaging: From Nano to Macro, 2011.

[5] S. H Joshi, E. Klassen, A. Srivastava, and I. Jermyn. An efficient representation for computing geodesics between n-dimensional elastic shapes. In Proc. IEEE Conference on Computer Vision and Pattern Recognition, 2007.

[6] W. Liu, A. Srivastava, and E. Klassen. Joint shape and texture analysis of objects boundaries in images using a riemannian approach. In Proc. Asilomar Conference on Signals, Systems, and Computers, 2008.

[7] G. J. Bassell and S. T. Warren. Fragile x syndrome: loss of local mrna regulation alters synaptic development and function. Neuron, 60(2):201-214, 2008.

[8] M. Pawlik and N. Augsten. Rted: A robust algorithm for the tree edit distance. In PVLDB 5(4):334-345, 2011. 